\title{
AN EVOLUTIONARY GAME-THEORETIC APPROACH FOR IMAGE INTERPOLATION
}

\author{
Yan Chen, Yang Gao, and K. J. Ray Liu \\ Dept. ECE, University of Maryland College Park \\ E-mail:\{yan, yanggao, and kjrliu\}@umd.edu
}

\begin{abstract}
In this paper, we study the image interpolation from the game theoretic perspective and formulate the image interpolation problem as an evolutionary game. In this evolutionary game, the players are the unknown high resolution pixels and the pure strategies of the players are the corresponding low resolution neighbors. By regarding the non-negative weights of the low resolution pixels as the probabilities of selecting the pure strategies, the problem of estimating the high resolution pixels becomes finding the evolutionarily stable strategies for the evolutionary game. Experimental results show that the proposed game theoretical approach can achieve better performance than the state-of-the-art image interpolation methods in terms of both PSNR and visual quality.
\end{abstract}

Index Terms - Image interpolation, game theory, evolutionary game.

\section{INTRODUCTION}

One of the most important tasks in the field of image processing is spatial resolution up-conversion. Image interpolation is the technique addressing the problem of spatial resolution up-conversion. It generates a high resolution image from the input low resolution image by exploiting the inherent relationship between them.

The common image interpolation methods are the conventional linear interpolation schemes such as bilinear and bicubic interpolation [1]. These methods generate the high resolution image using a spatial-invariant linear interpolation filter. Although the computational complexity is low, these methods are not favored since they introduce a lot of blurring and ringing artifacts.

To overcome the drawbacks of conventional linear interpolation schemes, many more sophisticated adaptive image interpolation methods have been proposed. Jensen and Anastassiou proposed to first detect edges and then fit them with some templates to improve the interpolation result [2], while Carrato and Tenze optimized the interpolation parameters by using some predetermined edge pattern [3]. Based on the assumption that the covariance matrix of the high resolution image can be well estimated from the covariance matrix of the low resolution image, $\mathrm{Li}$ and Orchard proposed an edge-

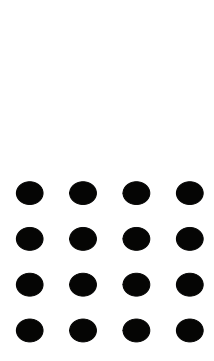

(a)

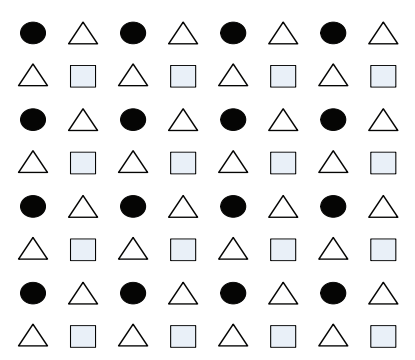

(b)
Fig. 1. Image interpolation: (a) the low resolution image that is directly down-sampled from the high resolution image; (b) the high resolution image, where the pixels denoted by square and triangle are the unknown high resolution pixels to be estimated.

preserved interpolation scheme [4]. For better interpolation, Zhang and Wu proposed to first generate multiple reconstructions from different directions, and then fuse the results by minimum mean square error estimation [5]. To further improve the interpolation results, a soft-decision adaptive interpolation (SAI) technique is proposed in [6] by combining the piecewise 2-D autoregressive modelling and block estimation.

In this paper, we revisit the image interpolation from the game theoretic perspective and formulate the image interpolation problem as an evolutionary game. In this evolutionary game, every unknown high resolution pixel is treated as an individual player, and their pure strategies are the neighboring low resolution pixels. The objective of the player is to find the evolutionarily stable strategy, i.e., the optimal combination of the low resolution pixels, to achieve better interpolation performance.

The rest of this paper is organized as follows. We give an introduction about evolutionary game model in section 2 . Then in section 3, we describe in details how to formulation the image interpolation problem as an evolutionary game, and discuss how to choose the pure strategy set and how to define the payoff function. Experimental results are shown in section 4. Finally, we draw conclusions in section 5 . 


\section{EVOLUTIONARY GAME MODEL}

A game $\mathbf{G}=\{U, A, F\}$ is generally defined as follows. $U=$ $\left\{u_{1}, u_{2}, \ldots, u_{N}\right\}$ are the players who play the game. $\mathbf{A}=$ $A_{1} \times A_{2} \times \ldots \times A_{N}$ are the pure strategy sets, where $A_{i}$ is the pure strategy set containing all possible pure strategies for user $u_{i}$. Let $a_{i} \in A_{i}$ be one possible strategy for $u_{i}$, then $\mathbf{a}=\left(a_{1}, a_{2}, \ldots, a_{N}\right) \in \mathbf{A}$ is a strategy profile of $\mathbf{U}$. $\mathbf{F}=\left\{f_{1}, f_{2}, \ldots, f_{N}\right\}$ are the players' payoff/utility functions, where $f_{i}$ is the payoff function of $u_{i}$. In general, $f_{i}$ is determined by all players' strategies a rather than $a_{i}$ only, i.e., $f_{i}=f_{i}(\mathbf{a})$. Let $a_{-i}=\left(a_{1}, \ldots, a_{i-1}, a_{i+1}, \ldots, a_{N}\right)$ be the players' strategy profile except player $u_{i}$.

Besides pure strategies, players can also take a mixed strategy by randomizing among different pure strategies. Suppose there are $M_{i}$ pure strategies in the pure strategy set $A_{i}=\left\{a_{i}^{1}, \ldots, a_{i}^{M_{i}}\right\}$, and let $p_{i j}, j=1, \ldots, M_{i}$ be the probability of $u_{i}$ choosing the $j^{\text {th }}$ pure strategy $a_{i}^{j}$, then $\mathbf{p}_{i}=\left(p_{i 1}, \ldots, p_{i M_{i}}\right)$ is a mixed strategy.

Since the payoff function $f_{i}$ is generally not only determined by $a_{i}$ but also by $a_{-i}$, to maximize the payoff, every player needs to know all other players' strategies. However, due to selfish nature, players will cheat if cheating can improve their payoffs, which means that all players are uncertain of other player strategies and payoffs. In such a case, to improve their payoffs, players will try different strategies in every play and learn from the strategic interactions using the methodology of understanding-by-building, which leads to the concept of "Evolutionary Game" [7].

An evolutionary game is a game that studies the evolution of the interaction dependent strategy in populations. One key concept in evolutionary games is the evolutionarily stable strategy (ESS), which is "a strategy such that, if all members of the population adopt it, then no mutant strategy could invade the population under the influence of natural selection". To study how the strategies spread over the population by natural selection, we adopt the Wright-Fisher model [8], which is by far the most popular stochastic model for reproduction in population genetics. It is based on the assumption that the probability of an individual adopting a certain strategy is proportional to the expected payoff of the population using that strategy. Therefore, the strategy spreading equation can be written as

$$
p_{i j}^{t+1}=\frac{p_{i j}^{t} f_{i}^{t}\left(a_{i}^{j}, a_{-i}\right)}{\sum_{k=1}^{M_{i}} p_{i k}^{t} f_{i}^{t}\left(a_{i}^{k}, a_{-i}\right)},
$$

where the numerator $p_{i j}^{t} f_{i}^{t}\left(a_{i}^{j}, a_{-i}\right)$ is the expected payoff of $u_{i}$ using strategy $a_{i}^{j}$, and the denominator $\sum_{k=1}^{M_{i}} p_{i k}^{t} f_{i}^{t}\left(a_{i}^{k}, a_{-i}\right)$ is the total expected payoff of $u_{i}$ using different strategies, which is the normalization term that ensures $\sum_{j=1}^{M_{i}} p_{i j}^{t+1}=1$.

\section{IMAGE INTERPOLATION AS AN EVOLUTIONARY GAME}

As in many previous papers, we assume that the low resolution image is directly downsampled from the high resolution image, i.e., $I_{l}(n, m)=I_{h}(2 n-1,2 m-1), 1 \leq n \leq N_{H}, 1 \leq$ $m \leq M_{W}$. As shown in Figure 1, the essential problem of image interpolation is to estimate the unknown pixels in the high resolution image based on the low resolution image. Obviously, this problem is ill-posed. To find a good estimate for the unknown high resolution pixels, we need to exploit the correlation between the low resolution pixels and the high resolution pixels. One possible approach is to use the spatially varying linear filter, i.e., each unknown high resolution pixel can be estimated using weighted average of a set of neighboring low resolution pixels.

$$
\hat{I}_{h}(i, j)=\sum_{(k, l) \in \Omega_{i j}} w_{k l} I_{l}(k, l),
$$

where $\Omega_{i j}$ is the candidate set of neighboring low resolution pixels for $I_{h}(i, j)$ and $w_{k l}$ is the weight. Usually, we have the constraints that $0 \leq w_{k l} \leq 1$ and $\sum_{(k, l) \in \Omega_{i j}} w_{k l}=1$.

\subsection{Game Theoretic Formulation}

Now, let us formulate the image interpolation problem as an evolutionary game. We first match the variables in (2) to the components of an evolutionary game as follows.

- Players: unknown high resolution pixels $I_{h}(i, j)$

- Pure strategies: low resolution pixel $I_{l}(k, l)$

- Pure strategy set: the candidate set $\Omega_{i j}$

- Mixed strategy: the estimate $\hat{I}_{h}(i, j)$

- Probabilities in the mixed strategy: the non-negative normalized weights $w_{k l}$

Based on the above variable matching, the problem of finding the estimate of the high resolution pixels becomes the problem of finding a good mixed strategy of the evolutionary game, which is the evolutionarily stable strategy (ESS).

\subsection{Pure Strategy Set $\Omega_{i j}$}

Similar to most of the previous approaches, we reconstruct the high resolution image in two steps. In the first step, we estimate the unknown high resolution pixels surrounded by four low resolution pixels, i.e., $I_{h}(2 n, 2 m)$. In the second step, with the help of reconstructed high resolution pixels $I_{h}(2 n, 2 m)$, we estimate other unknown high resolution pixels $I_{h}(2 n-1,2 m)$ and $I_{h}(2 n, 2 m-1)$.

\subsubsection{Pure Strategy Set for $I_{h}(2 n, 2 m)$}

In the first step of the interpolation, we estimate the unknown $I_{h}(2 n, 2 m)$ using 12 neighboring low resolution pixels and its 


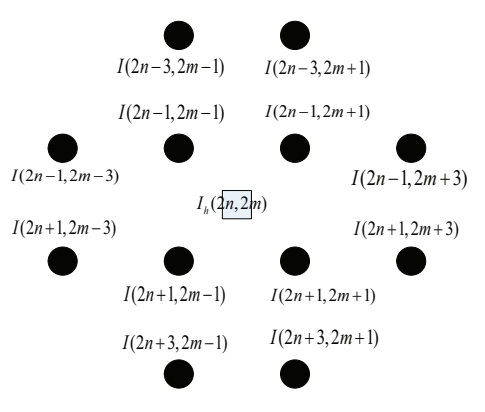

Fig. 2. The pure strategy set for $I_{h}(2 n, 2 m)$.

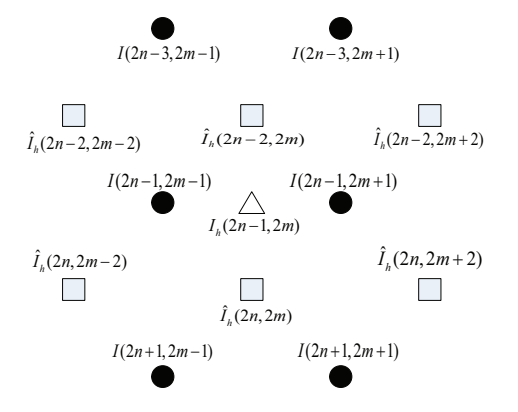

Fig. 3. The pure strategy set for $I_{h}(2 n-1,2 m)$.

previous estimate $\hat{I}_{h}^{t}(2 n, 2 m)$. Specifically, as shown in Figure 2, the pure strategy set for $I_{h}(2 n, 2 m)$ includes $I(2 n-$ $1,2 m-3), I(2 n-1,2 m+3), I(2 n-1,2 m-1), I(2 n-$ $1,2 m+1), I(2 n-3,2 m-1), I(2 n-3,2 m+1), I(2 n+$ $1,2 m-3), I(2 n+1,2 m+3), I(2 n+1,2 m-1), I(2 n+$ $1,2 m+1), I(2 n+3,2 m-1), I(2 n+3,2 m+1)$, and $\hat{I}_{h}^{t}(2 n, 2 m)$.

3.2.2. Pure Strategy Set for $I_{h}(2 n-1,2 m)$ and $I_{h}(2 n, 2 m-$ 1)

In the second step of the interpolation, we estimate the unknown $I_{h}(2 n-1,2 m)$ and $I_{h}(2 n, 2 m-1)$ using both the low resolution pixels and the estimate $\hat{I}_{h}(2 n, 2 m)$ in the first step. Specifically, as shown in Figure 3, the pure strategy set for $I_{h}(2 n-1,2 m)$ includes $I(2 n-1,2 m-1), I(2 n-1,2 m+1)$, $I(2 n-3,2 m-1), I(2 n-3,2 m+1), I(2 n+1,2 m-1), I(2 n+$ $1,2 m+1), \hat{I}_{h}(2 n-2,2 m), \hat{I}_{h}(2 n, 2 m), \hat{I}_{h}(2 n-2,2 m-2)$, $\hat{I}_{h}(2 n, 2 m-2), \hat{I}_{h}(2 n-2,2 m+2), \hat{I}_{h}(2 n, 2 m+2)$, and $\hat{I}_{h}^{t}(2 n-1,2 m)$. The pure strategy set for $I_{h}(2 n, 2 m-1)$ can be similarly generated.

\subsection{Payoff Function}

After choosing the pure strategy set, we now discuss how to define the payoff function. The payoff function $f_{i j}\left(a_{i j}, a_{-i j}\right)$ measure the player's payoff of taking strategy $a_{i j}$ when other players' strategies are $a_{-i j}$. Since the objective of the image interpolation problem is to recover the original high resolution image, the strategy that can lead to better reconstruction should have a higher payoff. With such an intuition, we define the payoff function as follows

$$
f_{i j}\left(a_{i j}, a_{-i j}\right)=\exp \left(-\frac{\left\|\mathrm{B}\left(a_{i j}\right)-\mathrm{B}\left(I_{h}(i, j)\right)\right\|^{2}}{\sigma^{2}}\right),
$$

where $\mathrm{B}(I(i, j))$ stands for the patch centered by $I(i, j)$ and $\sigma$ is a parameter.

From (3), we can see that if the pure strategy $a_{i, j}$ is more similar to the target pixel $\left.I_{h}(i, j)\right)$, the payoff is higher, which is reasonable since the pixels that are more similar to the target pixel should make more contribution during the interpolation process.

\section{EXPERIMENTAL RESULTS}

To evaluate the proposed game theoretical image interpolation method, we compare with the bicubic method and the soft-decision adaptive interpolation (SAI) method [6]. Two images: Lena and Boat, are tested.

The PSNR and visual quality comparison are shown in Figures 4 and 5. To better compare the details of the reconstructions among those three methods, we only illustrate the results of the upper-left region. As shown in Figure 4, (a) is the low resolution image that is directly down-sampled from the original high resolution Lena image; (b) is the interpolation result that is generated by the bicubic method, and the PSNR of the reconstruction is $36.89 \mathrm{~dB}$; (c) is the interpolation result that is obtained by the SAI method, where the PSNR of the reconstruction is $37.52 \mathrm{~dB}$; (d) is the reconstruction result of the proposed method, and the PSNR of the reconstruction is $38.04 \mathrm{~dB}$. We can see that the proposed game theoretical method can achieve the best PSNR performance. Moreover, by comparing (b) (c) and (d), we can see that bicubic introduces over-smooth artifacts and tends to blur the edge. With SAI method, the sharp edge can be well-preserved. However, at the same time, SAI may introduce some artificial edge distortion, e.g., the gray smooth region in (c). By finding the ESS for every player (unknown high resolution pixel), we can avoid the over-smooth artifacts and the artificial edge distortion, and thus obtain better reconstruction.

The results for the Boat image are shown in Figure 5. Similar to Figure 4 image, the proposed method achieves much better PSNR performance. Moreover, since the original Boat image contains some noise, the interpolation results generated by bicubic and SAI have severe visual distortions. However, with the proposed method, we can avoid such kinds of distortion and generate much better reconstruction. Therefore, with the proposed method, we are able to simultaneously perform interpolation and denoising.

\section{CONCLUSIONS}

In this paper, we investigated the image interpolation problem from a completely new angle: game theoretic perspective. 


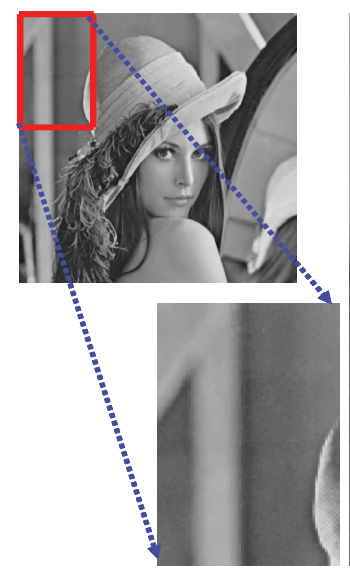

(a)

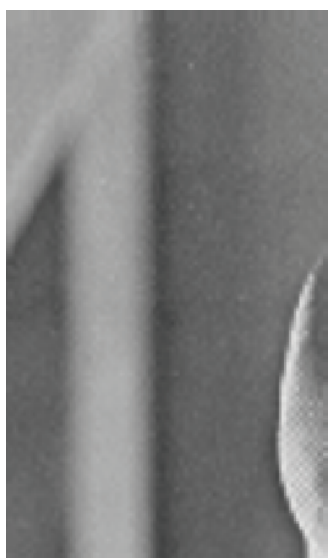

(b)

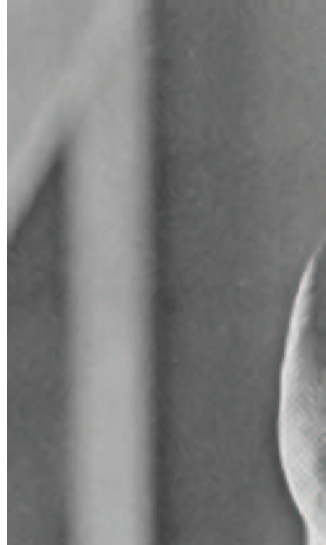

(c)

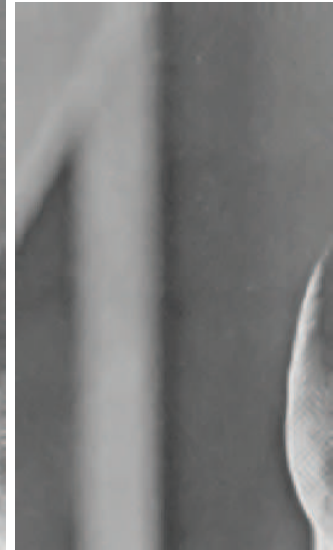

(d)

Fig. 4. The performance comparison for Lena: (a) the low resolution image; (b) the result generated by bicubic (36.89dB); (c) the result generated by the SAI method $(37.52 \mathrm{~dB})$; (d) the result generated by the proposed method $(38.04 \mathrm{~dB})$.

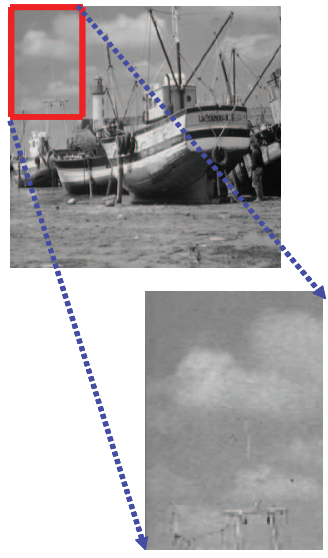

(a)

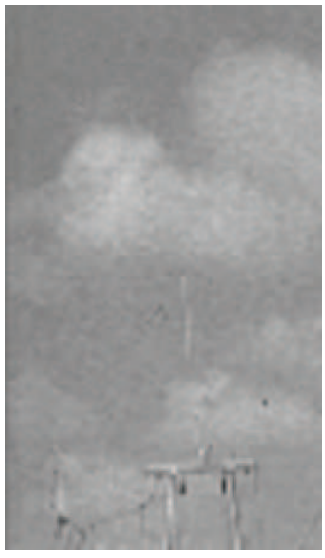

(b)

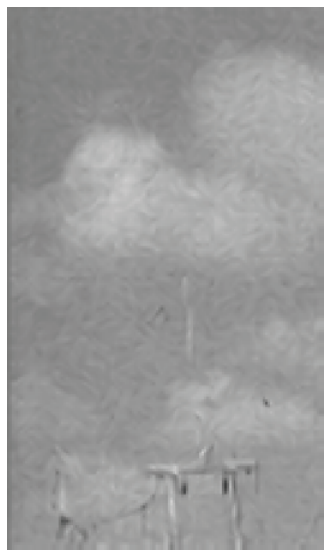

(c)

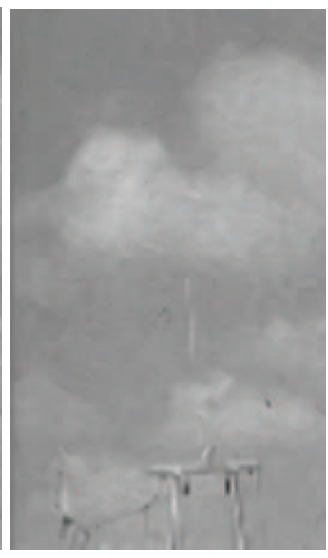

(d)

Fig. 5. The performance comparison for Boat: (a) the low resolution image; (b) the result generated by bicubic (32.73dB); (c) the result generated by the SAI method $(32.76 \mathrm{~dB})$; (d) the result generated by the proposed method $(33.55 \mathrm{~dB})$.

We treat each unknown high resolution pixel as an individual player and formulate the image interpolation as an evolutionary game. From such a perspective, the problem of estimating the high resolution pixels becomes finding the evolutionarily stable strategies for the evolutionary game. The experimental results show that compared with state-of-the-art image interpolation methods, the proposed game theoretical approach can achieve not only better PSNR performance but also better visual quality.

\section{REFERENCES}

[1] R. G. Keys, "Cubic convolution interpolation for digital image processing," IEEE TranS. Acoust., Speech, Signal Process., vol. 29, no. 6, pp. 1153-1160, Dec. 1981.

[2] K. Jensen and D. Anastassiou, "Subpixel edge localization and the interpolation of still images," IEEE Trans. Image Process., vol. 4, no. 3, pp. 285-295, Mar. 1995.
[3] S. Carrato and L. Tenze, "A high quality 2x image interpolator," IEEE Signal Process. Lett., vol. 7, no. 6, pp. 132-135, Jun. 2000.

[4] X. Li and M. T. Orchard, "New edge-directed interpolation," IEEE Trans. Image Process., vol. 10, no. 10, pp. 1521-1527, Oct. 2001.

[5] L. Zhang and X. Wu, "Image interpolation via directional filtering and data fusion," IEEE Trans. Image Process., vol. 15, no. 8, pp. 2226-2238, Aug. 2006

[6] X. Zhang and X. Wu, "Image interpolation by adaptive 2-d autoregressive modeling and soft-decision estimation," IEEE Trans. Image Process., vol. 17, no. 6, pp. 887-896, June 2008.

[7] Y. Chen, B. Wang, W.S. Lin, Y. Wu, and K.J.R. Liu, "Cooperative peerto-peer streaming: An evolutionary game-theoretic approach," to appear in IEEE Trans. on Circuits and Systems for Video Technology.

[8] R. Fisher, The genetical theory of natural selection, Clarendon Press, Oxford, 1930. 